\title{
Islanding Detection Method for Inverter-Based Distributed Generation by Injecting Second Order Harmonic Current
}

\author{
Byung-Moon Han* \\ Dept. of Electrical Engineering \\ Myongji University \\ Yongin, Korea \\ e-mail: erichan@mju.ac.kr
}

\begin{abstract}
This paper proposes a new islanding detection scheme for the inverter-based distributed generator by injecting negligible amount of the 2 nd order harmonic current. A proportional resonant controller was used for the output current control of the inverter, and a proportional resonant filter was used for extracting the 2nd order harmonic voltage at the point of common coupling (PCC). The islanding state can be detected by measuring the magnitude ratio of the 2 nd order harmonic voltage to the fundamental voltage when the 2 nd order harmonic current with $0.8 \%$ magnitude is injected.
\end{abstract}

\section{Key words}

Inverter-based distributed generation (DG), Islanding detection, 2nd order harmonic current injection, Proportional resonant (PR) controller, Proportional resonant filter

\section{Introduction}

Inverter-based distributed generation (DG) should be protected from the grid fault by fast disconnection and detection of the islanding state. When islanding occurs, the DG changes the operation from the current control to the voltage control. According to the international regulations such as UL1741 and IEEE1547, Islanding should be detected within 2 seconds [1]-[4].

This paper proposes an active islanding detection method for a DG unit. The proposed method is to inject the 2nd order harmonic current with $0.8 \%$ magnitude through the current controllers, and measuring the corresponding 2nd order harmonic voltage at the PCC. In order to measure the 2nd order harmonic voltage, the proportional resonant (PR) controller and PR filter were adopted [5], [6].

The magnitude of injected 2nd order harmonic current was determined to be $0.8 \%$ which is less than the allowable value of $1.08 \%$ in the IEC-61000 specification. The advantage of proposed method is to offer a simple, fast, and accurate islanding detection under the unbalanced grid voltage and the unbalanced load.

\section{Islanding Detection Scheme}

Fig. 1 shows a circuit diagram for the islanding detection described in UL1741. The AC grid, distributed power source, and load are assumed to be a lumped parameter model using RLC components. Here $\mathbf{R}_{\mathbf{f}}$ and $\mathbf{L}_{\mathbf{f}}$ represent resistance and inductance of the series harmonic filter. The transformer connects the distributed power source to the grid at the PCC. The load resonant frequency was set to $60 \mathrm{~Hz}$, which is same as the grid frequency.

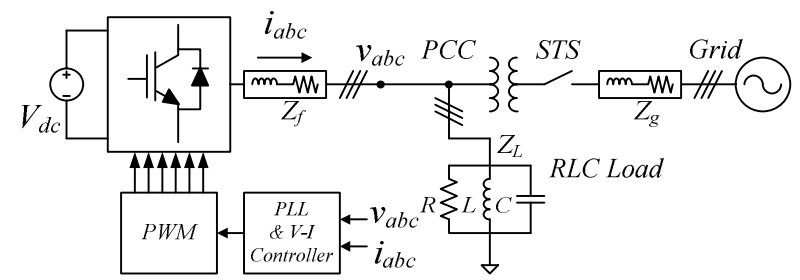

Fig. 1 UL1741 Standard Islanding Detection Circuit

Fig. 2 shows a circuit diagram of the grid-tied DG analyzed in this paper. The DG is connected to the AC grid through a harmonic filter and transformer.

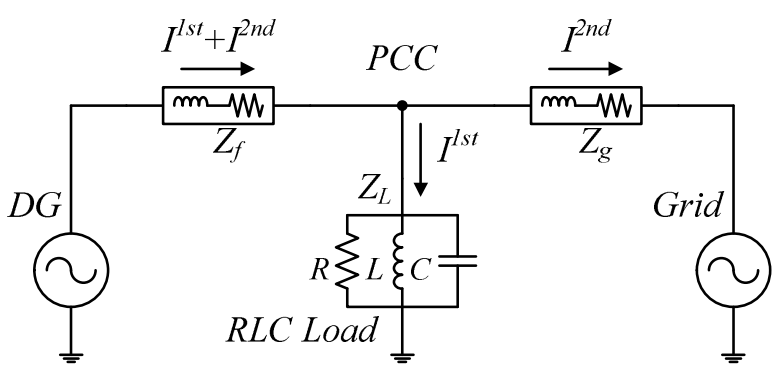

Fig. 2 Circuit Diagram for Grid-tied DG

In this circuit, $\mathbf{I}^{\text {sst }}$ represents the $60 \mathrm{~Hz}$ fundamental current and $\mathbf{I}^{\mathbf{2 n d}}$ represents the 2 nd order harmonic current. When the 2nd order harmonic current is injected from the DG, the grid impedance looks relatively smaller 
than the load impedance, so the 2nd order harmonic current flows into the grid entirely.

In the case where the DG is connected to the AC grid, the fundamental and the 2nd order harmonic voltages applied to the PCC can represented as shown by equations (1) and (2) respectively. Here, since the impedance values of $\mathbf{Z}_{\mathbf{g}}^{\mathbf{1 s t}}$ and $\mathbf{Z}_{\mathbf{g}}^{\mathbf{2 n d}}$ are much smaller than those of $\mathbf{Z}_{\mathbf{L}}^{\mathbf{1 s t}}$ and $\mathbf{Z}_{\mathbf{L}}^{\mathbf{2 n d}}$, it can be assumed that $\mathbf{Z}_{\mathbf{g}}^{\mathbf{1 s t}} \approx \mathbf{0}$ and $\mathbf{Z}_{\mathbf{g}}^{\mathbf{2 n d}} \approx \mathbf{0}$.

$$
\begin{aligned}
& V_{\text {pcc }}^{1 s t}=Z_{L}^{1 s t} \frac{V_{g}+I^{1 s t} Z_{g}^{1 s t}}{Z_{L}^{1 s t}+Z_{g}^{1 s t}} \approx V_{g} \\
& V_{\text {pcc }}^{\text {2nd }}=I^{2 n d} \frac{Z_{L}^{2 n d} Z_{g}^{2 n d}}{Z_{L}^{2 n d}+Z_{g}^{2 n d}} \approx 0
\end{aligned}
$$

However, if the static transfer switch (STS) is opened and the operation mode is changed to the islanding, the fundamental voltage and the 2 nd order harmonic voltage at the PCC can be expressed by equations (3) and (4)

$$
\begin{gathered}
V_{\mathrm{pcc}}^{1 \text { st }} \approx \mathbf{I}^{1 \mathrm{st}} \mathrm{Z}_{\mathrm{L}}^{1 \text { st }} \\
\mathbf{V}_{\mathrm{pcc}}^{\text {2nd }} \approx \mathbf{I}^{2 \mathrm{nd}} \mathrm{Z}_{\mathrm{L}}^{\text {2nd }}
\end{gathered}
$$

Where, $\mathbf{I}^{\mathbf{1 s t}}$ and $\mathbf{I}^{\mathbf{2 n d}}$ are the magnitude of the fundamental current and the 2nd order harmonic current injected by only the DG respectively.

When the 2nd order harmonic current is applied to the RLC load, the 2nd order harmonic voltage is visible at the PCC. This phenomenon can provide a new islanding detection scheme. This paper proposes a new islanding detection scheme by evaluating the magnitude ratio of the 2nd order harmonic voltage to the fundamental voltage in (5).

$$
\frac{V_{\text {pcc }}^{2 n d}}{V_{\text {pcc }}^{1 s t}}=\frac{I^{2 n d} Z_{L}^{2 n d}}{I^{1 s t} Z_{L}^{1 s t}}
$$

Fig. 3 shows the variation of the magnitude ratio of the 2nd order harmonic voltage to the fundamental voltage when injecting the 2nd harmonic current of $0.8 \%$ magnitude into the UL1741 test circuit. This voltage magnitude ratio is nearly zero before the STS opens at $1.0 \mathrm{~s}$ and up to $0.2 \%$ with a slope depending on the filter impedance on inverter and the load impedance.

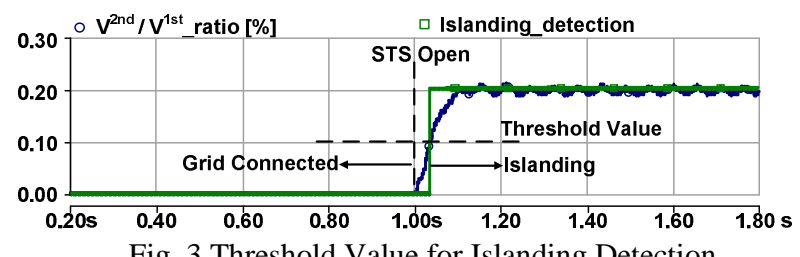

\section{2nd Order PR Controller}

Since the PR controller has no steady-state error, it provides a simple control on the stationary reference frame without complicated rotational reference frame transform. So, the control algorithm can be easily implemented with low-cost microprocessor. The PR controller can inject the 2nd order harmonic current with parallel resonant controller [25], [26]. The PR controller can be expressed by equation (8).

$$
G_{P R}^{\alpha \beta}=\left[\begin{array}{cc}
K_{p}+\frac{K_{i} s}{s^{2}+\omega^{2}} & 0 \\
0 & K_{p}+\frac{K_{i} s}{s^{2}+\omega^{2}}
\end{array}\right]
$$

Where, $\boldsymbol{\omega}$ is resonant frequency of controller, $\mathbf{K}_{\mathbf{p}}$ is proportional gain, and $\mathbf{K}_{\mathbf{i}}$ is integration gain.

Since the PR controller has an excellent performance to eliminate the steady-state error of the AC signal, the 2nd order harmonic current controller is connected in parallel with the fundamental current controller as shown in Fig. 4.

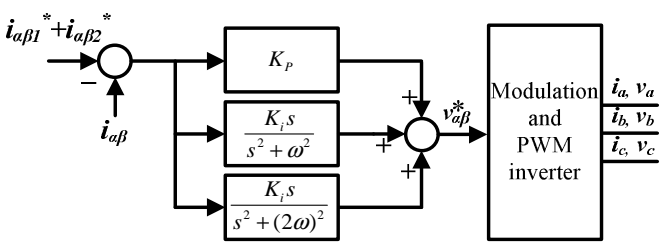

Fig. 4 Configuration of PR Current Controller

In this figure, $\mathbf{i}_{\boldsymbol{\alpha} \boldsymbol{\beta} \mathbf{1}}^{*}, \mathbf{i}_{\boldsymbol{\alpha} \boldsymbol{\beta} 2}^{*}$ are the reference values of the fundamental and the 2 nd order harmonic currents on the stationary reference frame, and $\mathbf{i}_{\boldsymbol{\alpha} \boldsymbol{\beta}}$ is the measured output current at the inverter output terminal.

Since the proposed scheme distinguishes the islanding state by the ratio of the 2 nd order harmonic voltage to the fundamental voltage, accurate measuring the magnitude of the fundamental voltage and the 2nd order harmonic voltage at the PCC is significant.

In order to implement the PR control in a digital system, appropriate discretization must be performed. A generalized integrator $(\mathrm{GI})$ can be transformed to the equivalent form (9).

$$
\frac{y(s)}{u(s)}=\frac{s}{s^{2}+\omega^{2}} \Leftrightarrow\left\{\begin{array}{c}
y(s)=\frac{1}{s}[u(s)-v(s)] \\
v(s)=\frac{1}{s} \cdot \omega^{2} \cdot y(s)
\end{array}\right.
$$

For the accurate measurement, a PR filter with a transfer function expressed in equation (10) was considered [27].

$$
G_{P R}(s)=\frac{k \omega_{r} s}{s^{2}+k \omega_{r} s+\omega_{r}^{2}}
$$

Where, $\boldsymbol{\omega}_{\mathbf{r}}$ is the resonant frequency of filter, $\mathbf{k}$ is the damping factor.

Since the fundamental and the 2nd order harmonic voltages are sinusoidal through filtering the inverter output voltage as shown in Fig. 5, the voltage magnitude ratio for islanding detection can be obtained by equation (11).

$$
\mathbf{V}_{\mathbf{p c c}}^{1 s t}=\sqrt{\left(\mathbf{v}_{\mathbf{f} \alpha}^{1 s t}\right)^{2}+\left(\mathbf{v}_{\mathbf{f} \beta}^{1 s t}\right)^{2}}
$$




$$
\mathbf{V}_{\mathrm{pcc}}^{2 \mathrm{nd}}=\sqrt{\left(\mathbf{v}_{\mathrm{f} \alpha}^{2 \mathrm{nd}}\right)^{2}+\left(\mathbf{v}_{\mathrm{f} \beta}^{2 n d}\right)^{2}}
$$

Where, $\mathbf{V}_{\mathbf{p c c}}^{\mathbf{1 s t}}$ and $\mathbf{V}_{\mathbf{p c c}}^{\mathbf{2 n d}}$ are the magnitudes of the fundamental voltage and the 2 nd order harmonic voltage.

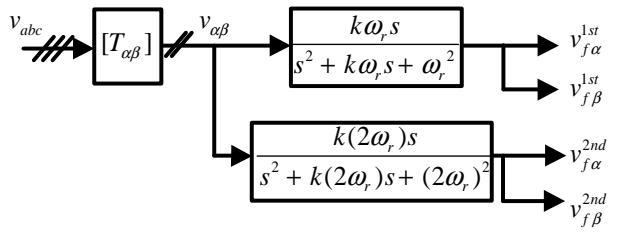

Fig. 5 Configuration of PR Filter

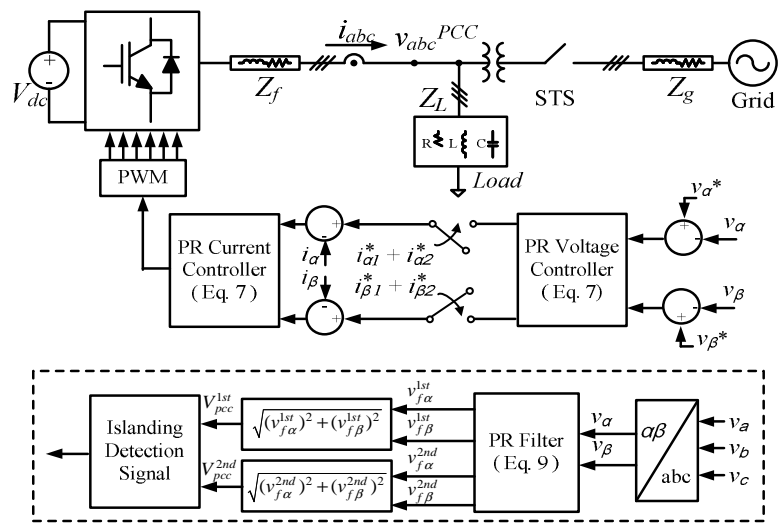

Fig. 6 Structure of Proposed Islanding Detection Scheme

Fig. 6 shows a block diagram to describe the proposed islanding detection method and the controller of inverter. After detecting the islanding state and opening the STS, the PR voltage controller, which is connected in series with the PR current controller, starts to maintain the PCC. So, the reference value of the PR current controller is determined by the output of the PR voltage controller, which has same structure represented in equation (8).

\section{Operation Analyses}

\section{A. Islanding detection under UL1741 conditions}

To verify the proposed islanding detection, simulations were conducted using the PSCAD/EMTDC. Table 1 show the circuit parameters of the inverter-based DG when the UL1741 regulation is applied. The inverter-based DG is represented by a battery energy storage system (BESS). $\mathbf{V}_{\text {grid }}$ and $\mathbf{f}_{\text {grid }}$ are the grid voltage and frequency. $\mathbf{L}_{\mathbf{f}}$ and $\mathbf{C}_{\mathbf{f}}$ are the filter inductor and capacitor and $\mathbf{R}_{\mathbf{f}}$ is a damping resistor for the filter. $\mathbf{Q}_{\mathbf{f}}$ is a quality factor and f_sw is a switching frequency. $\mathbf{R}_{\text {load }}, \mathbf{L}_{\text {load }}, \mathbf{C}_{\text {load }}$ are the RLC values of the load.

Fig. 7 shows the frequency variation at the PCC, the instantaneous grid current, the magnitude ratio of the 2 nd order harmonic voltage to the fundamental voltage and the islanding detection signal obtained from computer simulations with PSCAD/EMTDC. The power supplied by the BESS and the power consumption by the load were set to be equal.

The system is islanded at time $\mathrm{t}=1.0 \mathrm{~s}$ by opening STS. Prior to the islanding event, the BESS also injects 2nd order harmonic current through the PR current controller in addition to fundamental current. Since system frequency remains within the limit of $60 \pm 0.1 \mathrm{~Hz}$, the islanding state cannot be detected by sensing the frequency variation only.

\begin{tabular}{cc}
\multicolumn{2}{c}{ Table I. Test Circuit Parameters } \\
\hline Items & Parameter \\
\hline Rated Power & $5 \mathrm{~kW}$ \\
Battery Voltage & $336 \sim 470.4 \mathrm{~V}$ \\
$\mathbf{V}_{\text {grid }}$ & $220 \mathrm{Vrms}$ \\
Filter $\mathbf{L}_{\mathbf{f}}$ & $3 \mathrm{mH}$ \\
Filter $\mathbf{C}_{\mathbf{f}}$ & $15 \mathrm{uF}$ \\
$\mathbf{R}_{\mathbf{f}}$ & $0.1 \Omega$ \\
$\mathbf{f}_{\text {grid }}$ & $60 \mathrm{~Hz}$ \\
$\mathbf{f}_{\text {sw }}$ & $10 \mathrm{kHz}$ \\
$\mathbf{R}_{\text {load }}$ & $9.68 \Omega$ \\
$\mathbf{L}_{\text {load }}$ & $10.27 \mathrm{mH}$ \\
$\mathbf{C}_{\text {load }}$ & $685.1 \mathrm{uF}$ \\
\hline
\end{tabular}

Although the 2nd order harmonic current of $0.8 \%$ magnitude is injected by the PR current controller, the 2nd order harmonic voltage at the PCC is not visible until $\mathrm{t}=1.0 \mathrm{~s}$. However, the level of the $2 \mathrm{nd}$ order harmonic voltage appears while the 2 nd order harmonic current starts to flow into the load from 1.0s. Therefore, the magnitude ratio of the 2 nd order harmonic voltage to the fundamental voltage rises and reaches $0.2 \%$.

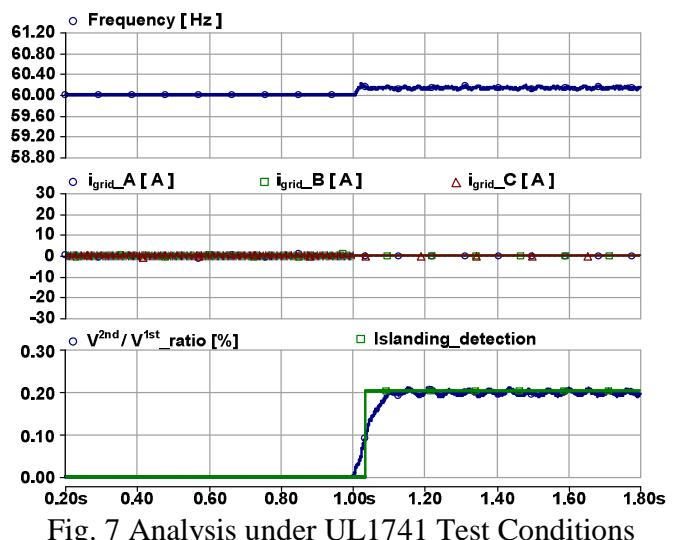

\section{B. Islanding detection under unbalanced grid voltage}

In actual distribution system, the grid voltage is not always balanced. If the instantaneous grid voltage is not balanced when the BESS is operated in coupled with the grid, a negative-sequence voltage will appear at the PCC. Because of this, islanding detection by injecting the negative-sequence current or voltage cannot be applied in general.

Fig. 8 shows the instantaneous grid voltage, the unbalance ratio, the magnitude ratio of the 2 nd order harmonic voltage to the fundamental voltage and the islanding detection signal. The top waveforms show the grid voltage in which Phase-B and -C voltages have $10 \%$ lower than Phase-A voltage.

The detection method based on a negative-sequence voltage injection cannot be used for islanding detection when the grid voltage unbalance is larger than $5 \%$. Therefore, grid unbalance ratio was set $10 \%$ and the 
islanding occurs at $1.0 \mathrm{~s}$ in this simulation. The magnitude ratio of the 2 nd order harmonic voltage to the fundamental voltage maintains at $0 \%$ under the voltage unbalance. However, when the islanding occurs, the magnitude ratio rises and reaches $0.2 \%$. So, the proposed scheme provides an accurate and fast islanding detection under the unbalanced grid voltage.

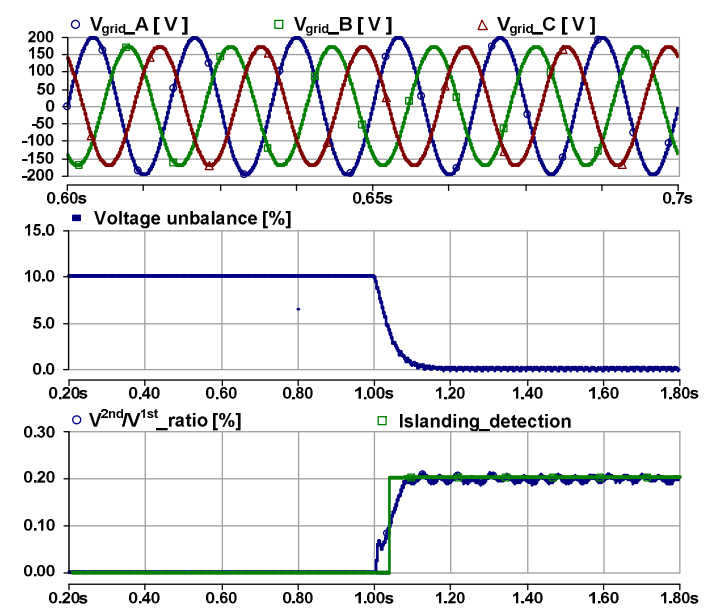

Fig. 8 Analysis under Unbalanced Grid Voltage

\section{Islanding detection under unbalanced load}

In this case study, all conditions comply with the UL1741 test condition except that the load resistance is not balanced. Simulation was performed to evaluate the islanding detection accuracy and the detection sensitivity due to the load unbalance. It is assumed that the load in phase-A is changed with a step variation of $\pm 10 \%$ between $0.2 \mathrm{~s}$ and $1.8 \mathrm{~s}$.

Fig. 9 shows the load current, the magnitude ratio of the 2nd order harmonic voltage to the fundamental voltage, and the islanding detection signal. Balanced three-phase load is operated between $0.2 \mathrm{~s}$ and $0.5 \mathrm{~s}$.

Unbalanced three-phase load reduced Phase-A load by $10 \%$ is operated between $0.5 \mathrm{~s}$ and $0.75 \mathrm{~s}$. Unbalanced three-phase load increased Phase-A load by $10 \%$ is operated between $0.75 \mathrm{~s}$ and $1.0 \mathrm{~s}$.

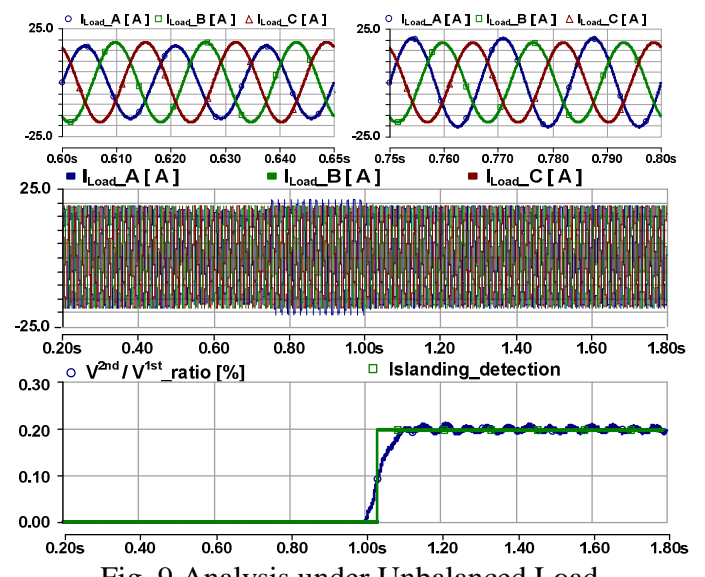

Fig. 9 Analysis under Unbalanced Load

The magnitude ratio of the 2 nd order harmonic voltage to the fundamental voltage maintains at $0 \%$ in spite of the load unbalance and the step variation. However, the voltage magnitude ratio rises and reaches $0.2 \%$ when islanding occurs at $1.0 \mathrm{~s}$. So, the voltage magnitude ratio can be used as a stable detection signal for islanding.

\section{Hardware Experiments}

Fig. 10 shows a hardware test set-up to verify the proposed islanding detection experimentally. The entire system was configured using a $5 \mathrm{kWh}$ lithium-polymer battery, a 5kVA Inverter, a main controller, an RLC load, and a $5 \mathrm{kVA}$ power transformer. To verify the validity of the simulations, all conditions for the experiment were set identically to those set forth in Table 1 .

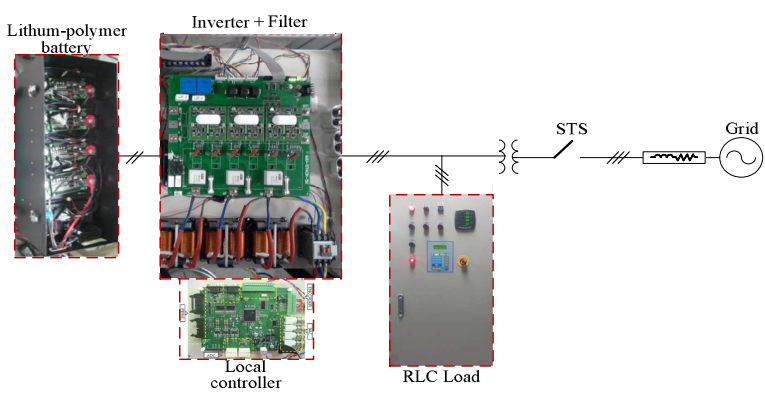

Fig. 10 Experimental Setup for Islanding Detection

Fig. 11 shows experimental results for islanding detection when the grid voltage is balanced. From the top, the figures show the variations of system frequency, the instantaneous grid current, voltage ratio, and islanding detection signal. Before the occurrence of islanding at $1.0 \mathrm{~s}$, small amounts of current flow into the grid because the power consumption of the load and the amount of power supplied by the BESS are the same. As the results in the simulation, frequency variation cannot be used for islanding detection because the frequency variation is negligible.

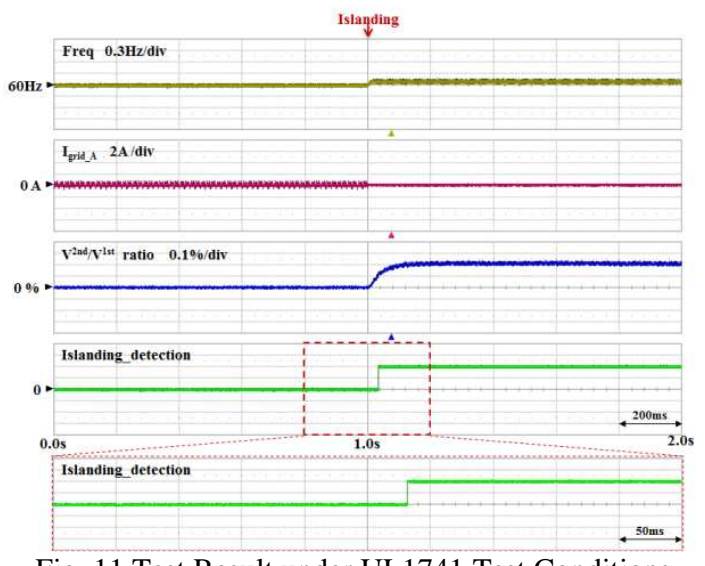

Fig. 11 Test Result under UL1741 Test Conditions

However, since the 2nd order harmonic current of $0.8 \%$ magnitude is injected after the occurrence of islanding, the grid is blocked and the level of 2 nd order harmonic voltage measured at the PCC rises simultaneously. However, the voltage magnitude ratio rises slowly and reaches $0.2 \%$. So, this value can be utilized to detect the islanding state.

Fig. 12 shows experimental results for islanding detection when the grid voltage is unbalanced. The unbalanced ratio was set $10 \%$ same as in the simulation. The voltage ratio of the 2 nd order harmonic voltage to the 
fundamental voltage does not increase before $1.0 \mathrm{~s}$. However, once the islanding occurs, the 2nd order harmonic voltage at the PCC appears due to the injected 2 nd order harmonic current. So, the voltage ratio of the 2nd order harmonic voltage to the fundamental voltage increases rapidly. The proposed method can effectively detect the islanding state under the unbalanced grid voltage.

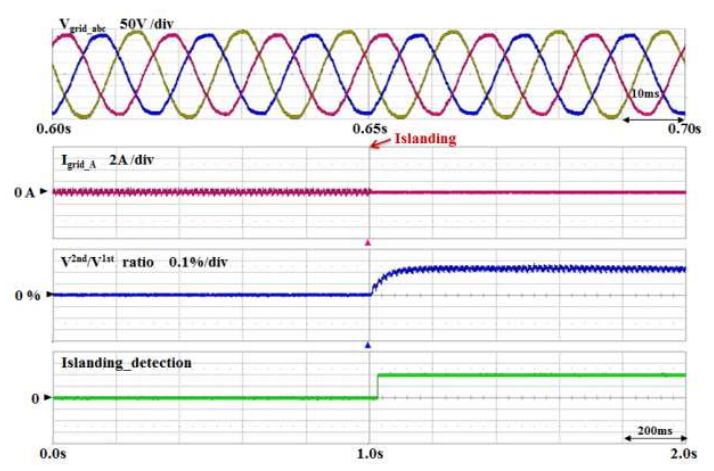

Fig. 12 Test Result under Unbalanced Grid Voltage

Fig. 13 shows experimental results for islanding detection when the load is unbalanced. The unbalanced ratio was set same as in the simulation. From $0.2 \mathrm{~s}$ to $0.6 \mathrm{~s}$ the load in one phase was increased by $10 \%$ of the rated power, and from $0.6 \mathrm{~s}$ to $2.0 \mathrm{~s}$ the load in one phase was decreased by $10 \%$ of the rated power. The voltage ratio of the 2 nd order harmonic voltage to the fundamental voltage under the load unbalance is maintained at $0 \%$ before the islanding occurs. After islanding occurs at $1.0 \mathrm{~s}$, the voltage ratio increases proportional to the magnitude of injected 2nd order harmonic current. The proposed method can effectively detect the islanding state under the unbalanced load voltage.

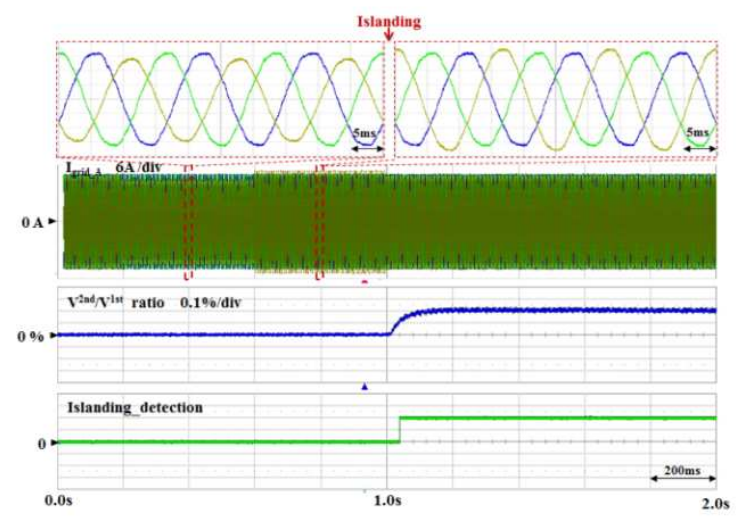

Fig. 13 Test Result under Unbalanced Load

\section{Conclusion}

This paper proposes a new islanding detection method by measuring the magnitude ratio of the 2 nd order harmonic voltage to the fundamental voltage at the PCC after injecting $0.8 \%$ of the 2 nd order harmonic current.

In order to measure the 2 nd order harmonic voltage and the fundamental voltage, proportional resonant (PR) controller and PR filter were adopted. The proposed method was verified through simulations with PSCAD/EMTDC.
The proposed method can offer the islanding detection time within $25 \mathrm{~ms}$ that is much faster than the international standard of $2.0 \mathrm{~s}$.

\section{Acknowledgement}

This research was supported by Korea Electric Power Corporation. (grant number : R18XA06-52)

\section{References}

[1] Liu F, Kang Y, Zhang Y, et al. "Improved SMS islanding detection method for grid-connected converters," Renewable Power Generation, 4(1): pp. 36-42, 2010.

[2] Youngseok Jung, Jaeho Choi, and G. Yu, "A Novel Active Anti-islanding Method for Grid-connected Photovoltaic Inverter," Journal of Power Electronics, Vol. 7, No. 1 p. 6471, 2007.

[3] Zhihui Dai; Zhiqiang Chong; Xuan Liu; Chuan Li, "Active islanding detection method based on grid-connected photovoltaic inverter and negative sequence current injection," Power System Technology (POWERCON), 2014 International Conference on , pp.1685,1690, 20-22 Oct. 2014

[4] H. Karimi, A. Yazdani and R. Iravani, "Negative-Sequence Current Injection for Fast Islanding Detection of a Distributed Resources," IEEE Trans. on Power Electronics, vol. 23, no. 1, Jan. 2008.

[5] D. Reigosa, F. Briz, C. Charro, P. Garcia, and M. Guerrero, "Active Islanding Detection Using High-Frequency Signal Injection," IEEE Trans. on Industry Application, vol. 48, no. 5, pp. 1588-1597, Sept./Oct. 2012.

[6] R. Teodorescu and F. Blaabjerg, "Proportional-resonant controllers. A new breed of controllers suitable for gridconnected voltage-source converters," in Proc. OPTIM, 2004, vol. 3, pp. 9-14. 